\title{
CONTINUOUS DEPENDENCE FOR SOME FUNCTIONAL DIFFERENTIAL EQUATIONS
}

\author{
YOSHIYUKI HINO*) \\ (Received on May 19, 1971)
}

Several authors have discussed global behaviors of trajectories of functional differential equations with the phase space considered by Hale ([2], [3], [4], [5]). The purpose of this paper is to discuss the continuity of solutions on initial values.

Let $x$ be any vector in $R^{n}$ and let $|x|$ be any norm of $x$. Let $B=$ $B\left((-\infty, 0], R^{n}\right)$ be a Banach space of functions mapping $(-\infty, 0]$ into $R^{n}$ with norm $\|\cdot\|$. For any $\phi$ in $B$ and any $\sigma$ in $[0, \infty)$, let $\phi^{\circ}$ be the restriction of $\phi$ to the interval $(-\infty,-\sigma]$. This is a function mapping $(-\infty,-\sigma]$ into $R^{n}$. We shall denote by $B^{o}$ the space of such functions $\phi^{\sigma}$. For any $\eta \in B^{o}$, we define the semi-norm $\|\eta\|_{B^{\circ}}$ of $\eta$ by

$$
\|\eta\|_{B^{\sigma}}=\inf _{\phi}\left\{\|\varphi\|: \varphi^{\sigma}=\eta\right\} .
$$

Then we can regard the space $B^{\sigma}$ as a Banach space with norm $\|\cdot\|_{B^{\sigma}}$. If $x$ is a function defined on $(-\infty, a)$, then for each $t$ in $(-\infty, a)$ we define the function $x_{t}$ by the relation $x_{t}(s)=x(t+s),-\infty<s \leqq 0$. For numbers $a$ and $\tau, a>\tau$, we denote by $A_{\tau}{ }^{a}$ the class of functions $x$ mapping $(-\infty, a)$ into $R^{n}$ such that $x$ is a continuous function on $[\tau, a)$ and $x_{\varepsilon} \in B$. The space $B$ is assumed to have the following properties :

(I) If $x$ is in $A_{\tau}{ }^{a}$, then $x_{t}$ is in $B$ for all $t$ in $[\tau, a)$ and $x_{t}$ is a continuous function of $t$, where $a$ and $\tau$ are constants such that $\tau<a \leqq \infty$.

(II) All bounded continuous functions mapping $(-\infty, 0]$ into $R^{n}$ are in $B$.

(III) If a sequence $\left\{\boldsymbol{\varphi}_{k}\right\}, \boldsymbol{\Phi}_{k} \in B$, is uniformly bounded on $(-\infty, 0]$ with respect to norm $|\cdot|$ and converges to $\phi$ uniformly on any compact subset of $(-\infty, 0]$, then $\phi \in B$ and $\left\|\varphi_{k}-\phi\right\| \rightarrow 0$ as $k \rightarrow \infty$.

(IV) There are continuous, nondecreasing and nonnegative functions $b(r), c(r)$ defined on $[0, \infty), b(0)=c(0)=0$, such that

$$
\|\varphi\| \leqq b\left(\sup _{-\sigma \leqq s \leqq 0}|\varphi(s)|\right)+c\left(\left\|\varphi^{\sigma}\right\|_{B^{\sigma}}\right)
$$

*) Supported in part by the Sakkokai Foundation. 
for any $\phi$ in $B$ and any $\sigma \geqq 0$.

(V) If $\sigma$ is a nonnegative number and $\phi$ is an element in $B$, then $T_{\sigma} \phi$ defined by $T_{o} \varphi(s)=\varphi(s+\sigma), s \in(-\infty,-\sigma]$, is an element in $B^{\circ}$.

(VI) $|\varphi(0)| \leqq M_{1}\|\varphi\|$ for some constant $M_{1}>0$.

(VII) $\left\|T_{t} \varphi\right\|_{B^{t}} \leqq M_{2}\|\varphi\|$ for all $t \geqq 0$ and for some constant $M_{2}>0$.

REMARK 1. When we discussed the global behaviors of trajectories in the phase space, the property of the fading memory, that is, $\left\|T_{\circ} \varphi\right\|_{B^{a}} \rightarrow 0$ as $\sigma \rightarrow \infty$, played an important role, but in this paper, this property is not required.

REMARK 2. The class of phase spaces considered by Coleman and Mizel [1] has the properties $(\mathrm{I}) \sim(\mathrm{VII})$, and hence the result in this paper holds good for this class of phase spaces.

Consider the functional differential equations

$$
\dot{x}(t)=f\left(t, x_{t}\right) .
$$

The superposed dot denotes the right-hand derivative and $f(t, \varphi)$ is a continuous function of $(t, \varphi)$ which is defined on $I \times B^{*}$ and takes values in $R^{n}$, where $I$ and $B^{*}$ are open subsets of $[0, \infty)$ and $B$, respectively. We shall denote by $x\left(t_{0}, \varphi\right)$ a solution of (1) such that $x_{t_{0}}\left(t_{0}, \varphi\right)=\varphi$ and denote by $x\left(t, t_{0}, \varphi\right)$ the value at $t$ of $x\left(t_{0}, \varphi\right)$.

THEOREM. Suppose that a solution $u(t)=u\left(t, t_{0}, \phi^{0}\right),\left(t_{0}, \phi^{0}\right) \in I \times B^{*}$, of (1) defined on $\left[t_{0}, t_{0}+a\right]$ for some $a>0$ is unique for initial value problem. Then for any $\varepsilon>0$, there exists a $\delta(\varepsilon)>0$ such that if $(s, \psi) \in I \times B^{*},\left|s-t_{0}\right|<\delta(\varepsilon)$ and $\left\|\psi-\phi^{0}\right\|<\delta(\varepsilon)$, then $\left\|x_{t}(s, \psi)-u_{t}\left(t_{0}, \varphi^{0}\right)\right\|<\varepsilon$ for all $t \in\left[\max \left\{t_{0}, s\right\}, t_{0}+a\right]$, where $x(s, \psi)$ is a solution of (1) through $(s, \psi)$.

PRoOF. The set $\left\{u_{t}: t \in\left[t_{0}, t_{0}+a\right]\right\}$ is a compact subset of $B$, and hence there exists a positive number $d$ such that if $\left\|\rho-u_{t}\right\| \leqq d$ and $|s-t| \leqq d$, then $(s, \phi) \in I \times B^{*}$ for all $t \in\left[t_{0}, t_{0}+a\right]$, because $I \times B^{*}$ is an open subset. Since $f$ is continuous in $(t, \phi)$, we can assume that if $|t-s| \leqq d$ and $\left\|\varphi-u_{t}\right\| \leqq d$, then $\left|f(s, \varphi)-f\left(t, u_{t}\right)\right| \leqq 1$ for a $t \in\left[t_{0}, t_{0}+a\right]$. Thus it follows that

$$
|f(s, \varphi)| \leqq 1+\max \left\{\left|f\left(t, u_{t}\right)\right|: t \in\left[t_{0}, t_{0}+a\right]\right\},
$$

and hence there exists an $M>0$ such that $|f(s, \varphi)|<M$ on the set $D=\{(s, \varphi)$ : $\left.|s-t| \leqq d,\left\|\rho-u_{t}\right\| \leqq d, t \in\left[t_{0}, t_{0}+a\right]\right\}$. Moreover, there is a continuous function $g(t, \varphi)$ defined on $\left[t_{0}-d, t_{0}+a+2 d\right] \times B$ such that $|g|<M$ and 


$$
g(t, \phi)=f(t, \varphi) \quad \text { for }(t, \varphi) \in D \text {. }
$$

Clearly the solutions of

$$
\dot{y}(t)=g\left(t, y_{t}\right)
$$

are continuable to $t_{0}+a+2 d$.

Suppose that the conclusion of this theorem is false. Then there exists a positive number $\varepsilon_{0}, \varepsilon_{0}<d$, and sequences $\left\{\phi^{m}\right\},\left\{t_{m}\right\}$ and $\left\{\boldsymbol{\tau}_{m}\right\}$ such that $\left\|\boldsymbol{\varphi}^{m}-\boldsymbol{\varphi}^{0}\right\| \rightarrow 0$, $t_{m} \rightarrow t_{0}, \max \left\{t_{0}, t_{m}\right\}<\tau_{m} \leqq t_{0}+a$, and $\tau_{m} \rightarrow \tau_{0}$ as $m \rightarrow \infty$ and that

$$
\left\|x_{\tau_{m}}\left(t_{m}, \phi^{m}\right)-u_{\tau_{m}}\left(t_{0}, \phi^{0}\right)\right\|=\varepsilon_{0}
$$

and

$$
\left\|x_{t}\left(t_{m}, \varphi^{m}\right)-u_{t}\left(t_{0}, \varphi^{0}\right)\right\|<\varepsilon_{0} \quad \text { for } \max \left\{t_{m}, t_{0}\right\} \leqq t<\tau_{m} .
$$

For all sufficiently large $m$, the function $g^{m}(t, \varphi)$ given by

$$
g^{m}(t, \varphi)=g\left(t+t_{m}-t_{o}, \varphi\right)
$$

is defined on $\left[t_{0}, \tau_{0}+d\right] \times B$. Let $y\left(t, t_{m}, \varphi^{m}\right), t_{m} \leqq t \leqq t_{0}+a+d$, be a solution of (3) through $\left(t_{m}, \phi^{m}\right)$. Then $y^{m}(t)$ given by

$$
y^{m}(t)= \begin{cases}y\left(t+t_{m}-t_{0}, t_{m}, \varphi^{m}\right) & \text { for } t \in\left[t_{0}, \tau_{0}+d\right] \\ \varphi^{m}\left(t-t_{0}\right) & \text { for } t \in\left(-\infty, t_{0}\right)\end{cases}
$$

is a solution through $\left(t_{0}, \phi^{m}\right)$ of the functional differential equation

$$
\dot{y}(t)=g^{m}\left(t, y_{t}\right)
$$

We shall show that the sequence $\left\{y^{m}(t)\right\}$ is uniformly bounded and equi-continuous on the interval $\left[t_{0}, \tau_{0}+d\right]$ for all large $m$. For all large $m$ we have $\left|\phi^{m}(0)-\phi^{0}(0)\right|$ $\leqq M_{1}\left\|\phi^{m}-\varphi^{0}\right\| \leqq K$ for some constant $K>0$ by (VI), and hence

$$
\begin{aligned}
\left|y^{m}(t)\right| & \leqq\left|\phi^{m}(0)\right|+\int_{t_{0}}^{t}\left|g^{m}\left(s, y_{s}^{m}\right)\right| d s \\
& \leqq\left|\phi^{0}(0)\right|+K+M\left(\tau_{0}+d-t_{0}\right)
\end{aligned}
$$

Therefore $\left\{y^{m}(t)\right\}$ is uniformly bounded on $\left[t_{0}, \tau_{0}+d\right]$. For any $t_{1}, t_{2}, t_{0} \leqq t_{2}<t_{1}$ $\leqq \tau_{0}+d$, we have 


$$
\left|y^{m}\left(t_{1}\right)-y^{m}\left(t_{2}\right)\right| \leqq \int_{t_{2}}^{t_{1}}\left|g^{m}\left(s, y_{s}^{m}\right)\right| d s \leqq M\left(t_{1}-t_{2}\right)
$$

and hence $\left\{y^{m}(t)\right\}$ is equicontinuous on $\left[t_{0}, \tau_{0}+d\right]$. By Ascoli-Arzelà's Theorem, there exists a subsequence of $\left\{y^{m}(t)\right\}$ which converges to a function $y^{*}(t)$ uniformly on $\left[t_{0}, \tau_{0}+d\right]$. We shall denote it by $\left\{y^{m}(t)\right\}$ again. The limit function $y^{*}(t)$ is continuous and bounded on $\left[t_{0}, \tau_{0}+d\right]$.

Define $y(t)$ by

$$
y(t)=\left\{\begin{array}{lll}
y^{*}(t) & \text { for } & t \in\left[t_{0}, \tau_{0}+d\right] \\
\varphi^{0}\left(t-t_{0}\right) & \text { for } t \in\left(-\infty, t_{0}\right) .
\end{array}\right.
$$

Then $y_{t}$ belongs to $B$ for all $t \in\left[t_{0}, \tau_{0}+d\right]$, because $y\left(t_{0}\right)=\phi^{0}(0)$ and $y_{t} \in A_{t_{0}}^{\tau_{0}+d}$. We shall show that $y(t)$ is a solution of (3) through $\left(t_{0}, \phi^{0}\right)$.

First of all, we shall see that the set $S=\left\{y^{m}{ }_{s}: s \in\left[t_{0}, \tau_{0}+d\right], m\right.$; sufficiently large $\}$ is a relative compact subset of $B$. Take any sequence $\left\{\psi^{m}\right\}, \psi^{m} \in S$. Then, corresponding to each $m$, there are $k_{m}$ and $s_{m}$ such that $s_{m} \in\left[t_{0}, \tau_{0}+d\right]$, and $\psi^{m}=y^{k_{s_{m}}}$. If the set $\left\{k_{m} ; m=1,2, \cdots\right\}$ is finite, we can assume that $\psi^{m}=y^{k}{ }_{s_{m}}$ for a specified $k$. In this case, it is clear that there is a subsequence of $\left\{\psi^{m}\right\}$ which converges in $S$. In the case where the set $\left\{k_{m}\right\}$ is infinite, we can set $\psi^{m}=y^{m}{ }_{s_{m}}$. We can also assume that the sequence $\left\{y^{m}(t)\right\}$ converges to the function $y(t)$ uniformly on $\left[t_{0}, \tau_{0}+d\right]$. There exists an $s_{0}$ such that $s_{m} \rightarrow s_{0} \in\left[t_{0}, \tau_{0}+d\right]$ as $m \rightarrow \infty$. Define $z^{m}(t), \xi^{m}(t), z(t)$ and $\xi(t)$ by

$$
\begin{aligned}
z^{m}(t) & = \begin{cases}y^{m}(t) & \text { for } t \in\left[t_{0}, \tau_{0}+d\right] \\
\varphi^{m}(0) & \text { for } t \in\left(-\infty, t_{0}\right),\end{cases} \\
\xi^{m}(t) & = \begin{cases}0 & \text { for } t \in\left[t_{0}, \tau_{0}+d\right] \\
\varphi^{m}\left(t-t_{0}\right)-\phi^{m}(0) & \text { for } t \in\left(-\infty, t_{0}\right],\end{cases} \\
z(t) & =\left\{\begin{array}{lll}
y(t) & \text { for } t \in\left[t_{0}, \tau_{0}+d\right] \\
\varphi^{0}(0) & \text { for } t \in\left(-\infty, t_{0}\right)
\end{array}\right.
\end{aligned}
$$

and

$$
\xi(t)= \begin{cases}0 & \text { for } t \in\left[t_{0}, \tau_{0}+d\right] \\ \varphi^{0}\left(t-t_{0}\right)-\varphi^{0}(0) & \text { for } t \in\left(-\infty, t_{0}\right),\end{cases}
$$

respectively. For any $a \in R^{n}$, the symbol $\langle a\rangle$ will denote the constant function $\alpha$ such that $\alpha(s)=a$ for all $s \in(-\infty, 0]$. Since $y^{m}{ }_{s_{m}}=z^{m}{ }_{s_{m}}+\xi^{m}{ }_{s_{m}}$ and $y_{s_{0}}=z_{s_{0}}+\xi_{s_{0}}$, we have 
( 7 )

$$
\begin{aligned}
& \left\|y_{s_{m}}^{m}-y_{s_{0}}\right\|=\left\|z_{s_{m}}^{m}+\xi^{m}{ }_{s_{m}}-z_{s_{0}}-\xi_{s_{0}}\right\| \\
& \leqq\left\|z^{m}{ }_{s_{m}}-z_{s_{0}}\right\|+\left\|\xi^{m}{ }_{s_{m}}-\xi_{s_{0}}\right\| \\
& \leqq\left\|z_{s_{m}}^{m}-z_{s_{m}}\right\|+\left\|z_{s_{m}}-z_{s_{0}}\right\|+\left\|\xi_{s_{m}}^{m}-\xi_{s_{m}}\right\|+\left\|\xi_{s_{m}}-\xi_{s_{0}}\right\| \\
& \leqq b\left(\sup _{-\left(s_{m}-t_{0}\right) \leqq s \leqq 0}\left|y^{m}\left(s_{m}+s\right)-y\left(s_{m}+s\right)\right|\right) \\
& +c\left(\left\|<\phi^{m}(0)>^{s_{m}-t_{0}}-\left\langle\phi^{0}(0)>^{s_{m}-t_{0}} \|_{B^{s_{m}-t_{0}}}\right)+\right\| z_{s_{m}}-z_{s_{0}} \|\right. \\
& +b\left(\sup _{-\left(s_{m}-t_{0}\right) \leqq s \leqq 0}\left|\xi^{m}\left(s_{m}+s\right)-\xi\left(s_{m}+s\right)\right|\right) \\
& +c\left(\left\|T_{s_{m}-t_{0}} \xi_{t_{0}}^{m}-T_{s_{m}-t_{0}} \xi_{t_{0}}\right\|_{B^{s_{m}-t_{0}}}\right)+\left\|\xi_{s_{m}}-\xi_{s_{0}}\right\| .
\end{aligned}
$$

And hence, we have

$$
\begin{aligned}
\left\|y_{s_{m}}^{m}-y_{s_{0}}\right\| \leqq & b\left(\sup _{-\left(\tau_{0}+d-t_{0}\right) \leqq s \leqq 0} \mid y^{m}\left(\tau_{0}+d+s\right)-y\left(\tau_{0}+d+s\right) \|\right) \\
& +c\left(M_{2}\left\|<\phi^{m}(0)>-<\phi^{0}(0)>\right\|\right)+\left\|z_{s_{m}}-z_{s_{0}}\right\| \\
& +c\left(M_{2}\left\|\xi_{t_{0}}^{m}-\xi_{s_{0}}\right\|\right)+\left\|\xi_{s_{m}}-\xi_{s_{0}}\right\|
\end{aligned}
$$

because we have $0 \leqq s_{m}-t_{0} \leqq \tau_{0}+d-t_{0}$ and because $\xi^{m}(t)$ and $\xi(t)$ are identically zero on the interval $\left[t_{0}, s_{m}\right]$. Since $y^{m}(t)$ converges to the function $y(t)$ uniformly on $\left[t_{0}, \tau_{0}+d\right]$ as $m \rightarrow \infty$, the first term on the right-hand side of (8) tends to zero as $m \rightarrow \infty$. By (III), the second term also tends to zero, since $\left|\phi^{m}(0)-\phi^{0}(0)\right|$ $\leqq M_{1}\left\|\varphi^{m}-\phi^{0}\right\|$ by (IV). We have $z, \xi \in A_{t_{0}}^{\tau_{0}+d}$, and therefore the third term and the fifth term tend to zero as $m \rightarrow \infty$ by (I). The fourth term also tends to zero as $m \rightarrow \infty$ by (III), because

$$
\left\|\xi_{t_{0}}^{m}-\xi_{t_{0}}\right\| \leqq\left\|\phi^{m}-\phi^{0}\right\|+\left\|<\phi^{m}(0)>-<\phi^{0}(0)>\right\| .
$$

Thus we have

$$
\left\|y_{s_{m}}^{m}-y_{s_{0}}\right\| \rightarrow 0 \quad \text { as } m \rightarrow \infty
$$

which shows that $\bar{S}$ is a compact subset of $B$, where $\bar{S}$ is the closure of the set $S$.

Therefore $g^{m}(t, \phi)$ is a uniformly continuous function on $\left[t^{0}, \tau_{0}+d\right] \times \overline{S \text {. Since }}$ $y^{m}(t)$ is a solution of (6) through $\left(t_{0}, \phi^{m}\right)$, we have

$$
y^{m}(t)=\phi^{m}(0)+\int_{t_{0}}^{t} g^{m}\left(s, y^{m}{ }_{s}\right) d s
$$

for all $t \in\left[t_{0}, \tau_{0}+d\right]$. The left-hand side of (9) tends to $y(t)$ as $m \rightarrow \infty$. The first 
term on the right-hand side of (9) tends to $\varphi^{0}(0)$ as $m \rightarrow \infty$. Noting the uniform continuity of $g^{m}(t, \varphi)$ on $\left[t_{0}, \tau_{0}+d\right] \times \bar{S}$, we have

$$
\begin{gathered}
\lim _{m \rightarrow \infty}\left|\int_{t_{0}}^{t} g^{m}\left(s, y^{m}{ }_{s}\right) d s-\int_{t_{0}}^{t} g\left(s, y_{s}\right) d s\right| \\
\leqq \int_{t_{0}}^{t} \lim _{m \rightarrow \infty}\left|g^{m}\left(s, y^{m}{ }_{s}\right)-g\left(s, y_{s}\right)\right| d s \\
\leqq \int_{t_{0}}^{t} \lim _{m \rightarrow \infty}\left|g^{m}\left(s, y^{m}{ }_{s}\right)-g\left(s, y^{m}{ }_{s}\right)\right| d s \\
\quad+\int_{t_{0}}^{t} \lim _{m \rightarrow \infty}\left|g\left(s, y^{m}{ }_{s}\right)-g\left(s, y_{s}\right)\right| d s \\
=0,
\end{gathered}
$$

and hence the second term on the right-hand side of (9) tends to $\int_{t_{0}}^{t} g\left(s, y_{s}\right) d s$ as $m \rightarrow \infty$. Since $y^{m}{ }_{t_{0}} \rightarrow \phi^{0}$ as $m \rightarrow \infty, y(t)$ is a solution of (3) through $\left(t_{0}, \phi^{0}\right)$ which is defined on $t_{0} \leqq t \leqq \tau_{0}+d$, and hence $y(t)$ can be expressed by $y\left(t, t_{0}, \varphi^{0}\right)$.

$y\left(t, t_{m}, \phi^{m}\right)$ is clearly a solution of (1) through $\left(t_{m}, \phi^{m}\right)$ until $\left(t, y_{t}\left(t_{m}, \phi^{m}\right)\right)$ leaves the domain $D$ by (2). Since $\left(t, x_{t}\left(t_{m}, \phi^{m}\right)\right)$ belongs to $D$ on $\left[t_{m}, \tau_{m}\right]$ by (5), we can assume that

$$
y\left(t, t_{m}, \phi^{m}\right)=x\left(t, t^{m}, \phi^{m}\right)
$$

for $t \in\left[t_{m}, \tau_{m}\right]$. Thus clearly $y\left(t, t_{0}, \phi^{0}\right)$ is a solution of (1) through $\left(t_{0}, \phi^{0}\right)$ defined on $\left[t_{0}, \tau_{0}\right]$. On the other hand, we have

$$
\begin{aligned}
\| x_{\tau_{m}}\left(t_{m}, \phi^{m}\right)-u_{\tau_{m}}( & \left.t_{0}, \varphi^{0}\right) \| \\
= & \left\|y_{\tau_{m}}\left(t_{m}, \varphi^{m}\right)-u_{\tau_{m}}\left(t_{0}, \varphi^{0}\right)\right\| \\
= & \left\|y_{\tau_{m}+t_{0}-t_{m}}^{m}-u_{\tau_{m}}\left(t_{0}, \varphi^{0}\right)\right\| \\
\leqq & \left\|y_{\tau_{m}+t_{0}-t_{m}}^{m}-y_{\tau_{0}}\left(t_{0}, \varphi^{0}\right)\right\| \\
& \quad+\left\|y_{\tau_{0}}\left(t_{0}, \varphi^{0}\right)-u_{\tau_{0}}\left(t_{0}, \varphi^{0}\right)\right\| \\
& \quad+\left\|u_{\tau_{0}}\left(t_{0}, \varphi^{0}\right)-u_{\tau_{m}}\left(t_{0}, \varphi^{0}\right)\right\|,
\end{aligned}
$$

and hence it follows from (4) and (10) that 


$$
\begin{aligned}
\varepsilon_{0} \leqq & \left\|y_{\tau_{m}+t_{0}-t_{m}}^{m}-y_{\tau_{0}}\left(t_{0}, \varphi^{0}\right)\right\| \\
& +\left\|y_{\tau_{0}}\left(t_{0}, \varphi^{0}\right)-u_{\tau_{0}}\left(t_{0}, \varphi^{0}\right)\right\|+\left\|u_{\tau_{0}}\left(t_{0}, \varphi^{0}\right)-u_{\tau_{m}}\left(t_{0}, \varphi^{0}\right)\right\| .
\end{aligned}
$$

Taking sufficiently large $m$, we have $s_{m}=\tau_{m}+t_{0}-t_{m} \in\left[t_{0}, \tau_{0}+d\right]$ and $s_{m} \rightarrow \tau_{0}$ as $m \rightarrow \infty$, and hence the first term on the right-hand side of (11) tends to zero as $m \rightarrow \infty$, as in the calculation of (8). The third term on the right-hand side of (11) also tends to zero by (I). Thus we have

$$
\varepsilon_{0} \leqq\left\|y_{\tau_{0}}\left(t_{0}, \varphi^{0}\right)-u_{\tau_{0}}\left(t_{0}, \Phi^{0}\right)\right\|
$$

It follows from (III) that

$$
\begin{aligned}
\varepsilon_{0} & \leqq\left\|y_{\tau_{0}}\left(t_{0}, \varphi^{0}\right)-u_{\tau_{0}}\left(t_{0}, \varphi^{0}\right)\right\| \\
& \leqq b\left(\sup _{-\left(\tau_{0}-t_{0}\right) \leqq s \leqq 0}\left|y\left(\tau_{0}+s, t_{0}, \varphi^{0}\right)-u\left(\tau_{0}+s, t_{0}, \varphi^{0}\right)\right|\right),
\end{aligned}
$$

and hence there exists an $s^{*} \in\left[-\left(\tau_{0}-t_{0}\right), 0\right]$ such that

$$
\left|y\left(\tau_{0}+t^{*}, t_{0}, \phi^{0}\right)-u\left(\tau_{0}+s^{*}, t_{0}, \varphi^{0}\right)\right| \neq 0,
$$

which contradicts the uniqueness of the solution $u(t)$. This proves Theorem.

\section{REFERENCES}

[1] B. D. COlEmAN AND V. J. MIZEL, On the stability of solutions of functional differential equations, Arch. Rational Mech. Anal., 30(1968), 174-196.

[2] J. K. HALE, Dynamical systems and stability, J. Math. Anal. Appl., 26(1969), 39-59.

[3] Y. HINO, Asymptotic behavior of solutions of some functional differential equations, Tôhoku Math. J., 22(1970), 98-108.

[4] Y. HINO, On stability of the soluion of some functional differential equations, Funkcial. Ekvac., 14(1971), 47-60.

[5] T. NAITO, Integral manifolds for linear functional differential equations on some Banach space, Funkcial. Ekvac., 13(1970), 199-213. 
